\title{
Toll-like receptor 3 upregulation by type I interferon in healthy and scleroderma dermal fibroblasts
}

\author{
Sandeep K Agarwal ${ }^{1 *}$, Minghua Wu', Christopher K Livingston², Donald H Parks², Maureen D Mayes ${ }^{1}$,
} Frank C Arnett ${ }^{1}$, Filemon K Tan ${ }^{1}$

\begin{abstract}
Introduction: Increased levels of genes in the type I interferon (IFN) pathway have been observed in patients with systemic sclerosis (SSC), or scleroderma. How type I IFN regulates the dermal fibroblast and its participation in the development of dermal fibrosis is not known. We hypothesized that one mechanism by which type I IFN may contribute to dermal fibrosis is through upregulation of specific Toll-like receptors (TLRs) on dermal fibroblasts. Therefore, we investigated the regulation of TLR expression on dermal fibroblasts by IFN.

Methods: The expression of TLRs was assessed in cultured dermal fibroblasts from control and SSc patients stimulated with IFNa2. The ability of IFNa2 to regulate TLR-induced interleukin (IL)- 6 and CC chemokine ligand 2 production was also assessed. Immunohistochemical analyses were performed to determine whether TLR3 was expressed in skin biopsies in the bleomycin-induced skin fibrosis model and in patients with SSc.

Results: IFNa2 increased TLR3 expression on human dermal fibroblasts, which resulted in enhanced TLR3-induced IL-6 production. SSC fibroblasts have an augmented TLR3 response to IFNa2 relative to control fibroblasts. Pretreatment of fibroblasts with transforming growth factor (TGF) $\beta$ increased TLR3 induction by IFN $\alpha 2$, but coincubation of TGF- $\beta$ did not alter TLR3 induction by IFN. Furthermore, IFN $\alpha 2$ inhibits but does not completely block the induction of connective tissue growth factor and collagen expression by TGF- $\beta$ in fibroblasts. TLR3 expression was observed in dermal fibroblasts and inflammatory cells from skin biopsies from patients with SSc as well as in the bleomycin-induced skin fibrosis model.
\end{abstract}

Conclusions: Type I IFNs can increase the inflammatory potential of dermal fibroblasts through the upregulation of TLR3.

\section{Introduction}

Systemic sclerosis (SSc), or scleroderma, is a multisystem autoimmune disease clinically characterized by progressive fibrosis of the skin and internal organs. Pathologically, SSc exhibits three cardinal features: inflammation and autoimmunity, vasculopathy and excessive extracellular matrix (ECM) deposition [1]. The ECM consists of collagens, proteoglycans, fibrillins and other matrix molecules [2]. Located within this matrix are fibroblasts and myofibroblasts, key effectors of the fibrotic process. Resident and

\footnotetext{
* Correspondence: Sandeep.K.Agarwal@uth.tmc.edu

'Division of Rheumatology and Clinical Immunogenetics, Department of Internal Medicine, The University of Texas Health Science Center at Houston, 6431 Fannin Avenue, Houston, TX 77030, USA

Full list of author information is available at the end of the article
}

infiltrating cells in the dermis secrete soluble mediators, such as transforming growth factor $\beta$ (TGF- $\beta$ ), that activate fibroblasts and induce differentiation into myofibroblasts $[3,4]$. The myofibroblasts subsequently produce large amounts of ECM, leading to fibrosis. In addition to their role in ECM deposition, dermal fibroblasts and myofibroblasts are capable of secreting inflammatory cytokines and chemokines, such as interleukin (IL)- 6 and CC chemokine ligand 2 (CCL-2), important inflammatory mediators in SSc pathogenesis [5-8]. Thus, fibroblasts also may contribute to the development of dermal fibrosis through the production of these inflammatory mediators.

Current paradigms point toward systemic immune dysregulation as a central process that ultimately may 
lead to fibroblast activation. Biopsies of early SSc skin demonstrate perivascular infiltrates of mononuclear inflammatory cells, which produce cytokines and chemokines that recruit inflammatory cells and promote ECM deposition [9]. More recent studies in patients with SSc have identified dysregulation of type I interferon (IFN) pathways similar to those seen in patients with systemic lupus erythematosus (SLE) [10-12]. Gene expression profiling of peripheral blood has demonstrated the presence of a type I IFN signature in patients with SSc [12]. These findings have been confirmed in both circulating $\mathrm{CD} 14^{+}$monocytes and $\mathrm{CD} 4^{+} \mathrm{T}$-cells, as well as in skin biopsies from patients with SSc compared with healthy controls [13-15]. Together these data demonstrate the presence of a type I IFN signature in circulating blood cells and a major target organ (skin) in patients with SSc.

Type I IFNs are potent regulators of the immune system, where they modulate the differentiation, survival, proliferation and cytokine production of T-cells, B-cells and dendritic cells. Among the critical immunoregulatory functions of IFN is its ability to stimulate the expression of Toll-like receptors (TLRs) on dendritic cells. TLRs are a family of germ line-encoded proteins that serve as pattern recognition receptors capable of recognizing highly conserved motifs present in infectious microorganisms called pathogen-associated molecular patterns (PAMPs) [16]. While their roles are best characterized on antigen-presenting cells, various TLRs also are expressed on fibroblast populations $[17,18]$. Interestingly, IFN increases TLR3 and TLR7 expression on fibroblast-like synoviocytes (FLS) and enhances TLRinduced inflammatory cytokine production by FLS [18].

Given the reported influence of IFN on FLS and the importance of dermal fibroblasts in the pathogenesis of SSc, it is important to understand how IFN may modulate the dermal fibroblast. We hypothesized that one mechanism by which type I IFN may contribute to the pathogenesis of SSc is through upregulation of the expression of specific TLRs on dermal fibroblasts.

\section{Materials and methods Reagents}

Recombinant human TGF- $\beta$ and IFN $\alpha 2$ were purchased from eBioscience Inc. (San Diego, CA, USA). TLR agonists Pam3CysK4; polyinosinic:polycytidylic acid, or poly (I:C); lipopolysaccharide (LPS) and Gardiquimod ([1-(4amino-2-ethylaminomethylimidazo[4,5-c] quinolin-1-yl)2-methylpropan-2-ol]) were purchased from InvivoGen (San Diego, CA, USA).

\section{Fibroblast cultures}

Skin biopsy specimens of clinically uninvolved skin were obtained from patients with SSc and from control patients without a history of autoimmune disease. All patients with SSc fulfilled the American College of Rheumatology criteria for SSc [19]. All patients provided written consent, and the study was approved by the Committee for the Protection of Human Subjects at the University of Texas Health Science Center at Houston.

Dermal fibroblast cultures were isolated as previously described [20]. Cultured fibroblast strains were established by mincing tissues and placing them into 60 $\mathrm{mm}$ culture dishes secured by glass coverslips. The primary cultures were maintained in Dulbecco's modified Eagle's medium (DMEM), 10\% fetal bovine serum (FBS), $2 \mathrm{mM} \mathrm{L}$-glutamine, $100 \mathrm{U} / \mathrm{mL}$ penicillin, and 50 $\mu \mathrm{M}$ 2-mercaptoethanol at $37^{\circ} \mathrm{C}$ with $5 \% \mathrm{CO}_{2}$. Passages 4-8 dermal fibroblasts were used for experiments.

\section{RNA isolation and quantitative real-time polymerase chain reaction}

Fibroblasts $\left(3 \times 10^{4}\right)$ were cultured in $100 \mu \mathrm{L}$ DMEM with $10 \%$ FBS in 96-well plates overnight. Cultures were subsequently rested overnight in DMEM with bovine serum albumin (BSA), then stimulated with cytokines in DMEM with BSA for 24 hours. Total RNA was isolated and cDNA was synthesized using the TaqMan Gene Expression Cells-to-CT ${ }^{\mathrm{TM}}$ Kit (Applied Biosystems Inc., Foster City, CA, USA). Quantitative real-time PCR (qRTPCR) was performed using validated TaqMan Gene Expression assays for human TLR2 (Hs00152973_m1), TLR3 (Hs01551078_m1), TLR4 (Hs01060206_m1), TLR7 (Hs00152971_m1), TLR9 (Hs00152973_m1), connective tissue growth factor (CTGF) (Hs00170014_m1) and cyclophilin (Hs99999904_m1) (Applied Biosystems Inc.) on an Applied Biosystems 7900HT Fast Real-Time PCR System. Cyclophilin was used as an endogenous control to normalize transcription levels of total RNA in each sample. The data were analyzed using SDS 2.3 software (Applied Biosystems Inc., Foster City, CA, USA) and the comparative CT method $\left(2-{ }^{\Delta \Delta C}{ }_{\mathrm{T}}\right.$ method). The fold change was calculated as $2-{ }^{\Delta \Delta C} \mathrm{~T}$.

\section{Cytokine production}

Fibroblasts $\left(3 \times 10^{5}\right)$ were cultured in $1 \mathrm{ml}$ DMEM with $10 \%$ FBS in 24-well plates overnight. Cultures were subsequently rested overnight in DMEM with BSA, then stimulated with TLR agonists $(10 \mu \mathrm{g} / \mathrm{mL})$ in DMEM with BSA for 48 hours. Supernatants were harvested and frozen at $-80^{\circ} \mathrm{C}$. IL- 6 and CCL- 2 levels were determined by performing enzyme-linked immunosorbent assay (eBioscience, Inc.).

\section{Bleomycin dermal fibrosis mouse model}

Six- to eight-week-old female C57BL/6 mice (Jackson Laboratory, Bar Harbor, ME, USA) were used in these studies. The protocols were approved by the University 
of Texas Health Science Center at Houston Animal Care and Use Committee. Filter-sterilized bleomycin $0.02 \mathrm{U}$ per mouse was dissolved in phosphate-buffered saline (PBS) (Teva Parenteral Medicines, Irvine, CA, USA), or PBS was administered by daily subcutaneous injections for 28 days into the shaved backs of mice using a 27-gauge needle. At the end of the experiment, mice were humanely killed and lesional skin was processed for analysis.

\section{Immunohistochemistry}

Skin biopsies were obtained from four patients with SSc and from four healthy controls without a known history of autoimmune disease from the National Disease Research Interchange (Philadelphia, PA, USA). Fivemicrometer sections were deparaffinized, rehydrated and immersed in Tris-buffered saline and $0.1 \%$ Tween 20, then treated with target retrieval solution (Dako, Carpinteria, CA, USA) at $95^{\circ} \mathrm{C}$ for 10 minutes. Rabbit polyclonal primary antibodies against TLR3 or an isotype-matched control antibody (Abcam Inc., Cambridge, MA, USA) were used. Bound antibodies were detected using secondary antibodies from the Dako Cytomation Envision System-HRP (3,3-diaminobenzidine tetrahydrochloride). Sections were counterstained with hematoxylin.

\section{Statistical analysis}

Data were imported into GraphPad Prism software for graphing and analysis (GraphPad Software, Inc., La Jolla, CA, USA). Data are given as means, and error bars represent the standard error of the mean. Nonparametric paired (Mann-Whitney $U$ test) and unpaired (Wilcoxon signed-rank test) $t$-tests were used when appropriate.

\section{Results}

\section{TLR3 upregulation by IFN- $\alpha 2$ in cultured dermal}

\section{fibroblasts}

Dermal fibroblasts from controls were stimulated with media or human recombinant IFN $\alpha 2$ for 24 hours. Total RNA was isolated and qRT-PCR was performed to determine the relative expression of TLR2, TLR3, TLR4, TLR7, TLR8 and TLR9. As shown in Figure 1A, TLR3 expression was upregulated by IFNa2 (50$150 \mathrm{ng} / \mathrm{mL}$ ) at 6 hours and remained elevated at 24 and 48 hours. In contrast, TLR4 expression was slightly upregulated by IFN $\alpha 2$ at 6 hours, but at 24 and 48 hours no change in TLR4 expression was observed compared with dermal fibroblasts cultured in media alone. Expression of TLR2, TLR7, TLR8 and TLR9 was below the limits of detection (data not shown). Additional experiments demonstrated that TLR3 but not TLR4 expression was upregulated in a dose-dependent fashion
(Figure $1 \mathrm{~B}$ ), with a concentration as little as $1 \mathrm{ng} / \mathrm{mL}$ IFN $\alpha 2$ stimulating the expression of TLR3. These data clearly demonstrate the upregulation of TLR3 expression by IFNa2 in control dermal fibroblasts.

The upregulation of TLR3 expression by IFN $\alpha 2$ was compared between SSc and control dermal fibroblasts. The magnitude of induction of TLR3 expression by IFN $\alpha 2$ was significantly greater in dermal fibroblasts from patients with SSc than in controls (Figure 2A). This increase in TLR3 expression was observed when dermal fibroblasts were stimulated with IFN $\alpha 2$ at concentrations from 1 to $100 \mathrm{ng} / \mathrm{mL}$, although at $100 \mathrm{ng} / \mathrm{mL}$ the difference was not statistically significant (Figure 2B). These data demonstrate that SSc cultured fibroblasts have a greater magnitude of upregulation of TLR3 by IFN 2 than that of control fibroblasts.

\section{IFN $\alpha 2$ increases TLR3-induced IL-6 production in cultured dermal fibroblasts}

To determine whether the upregulation of TLR3 mRNA resulted in changes in functional TLR levels, dermal fibroblasts were preincubated with media alone or with $50 \mathrm{ng} / \mathrm{mL}$ IFN $\alpha 2$ for 24 hours. Cultures were subsequently stimulated with a panel of TLR agonists, and cytokine and chemokine production were assessed. $\mathrm{Pam}_{3} \mathrm{CysK}_{4}$ (a TLR2 agonist), poly(I:C) (a TLR3 agonist), LPS (a TLR4 agonist) and Gardiquimod (a TLR7/8 agonist) were all used at $10 \mu \mathrm{g} / \mathrm{mL}$ (Figure $3 \mathrm{~A}$ ).

Culture supernatants from control dermal fibroblasts stimulated with the TLR3 agonist poly(I:C) produced high levels of IL-6 and CCL-2. Preincubation of dermal fibroblasts with IFN 22 resulted in increased IL-6 production $(P=0.01)$ but not CCL-2 production compared with dermal fibroblasts preincubated with BSA. Consistent with the qRT-PCR data shown in Figure 1, preincubation with IFN 2 did not significantly increase TLR4-induced production of IL-6 or CCL-2. Last, while IFN $\alpha 2$ preincubation slightly increased the levels of IL-6 and CCL- 2 in cultures stimulated with TLR2 or TLR7/8 agonists, these levels were not higher than those of unstimulated dermal fibroblasts (data not shown). These data suggest that IFN 22 preincubation results in enhanced IL-6 production to the TLR3 agonist poly(I:C).

SSc dermal fibroblasts also demonstrated enhanced IL- 6 production to the TLR3 agonist poly(I:C), but not to other TLR agonists. In Figure 3B, the level of IL-6 in culture supernatants from cells preincubated with IFN 2 followed by TLR3 stimulation with poly(I:C) was significantly higher than that in SSc dermal fibroblasts preincubated in media alone followed by poly(I:C) stimulation $(P=0.002)$. In contrast, IFN 22 preincubation did not significantly increase poly(I:C)-induced production of CCL-2. The IL- 6 production in TLR2-stimulated cultures was not higher than that in media alone (data 


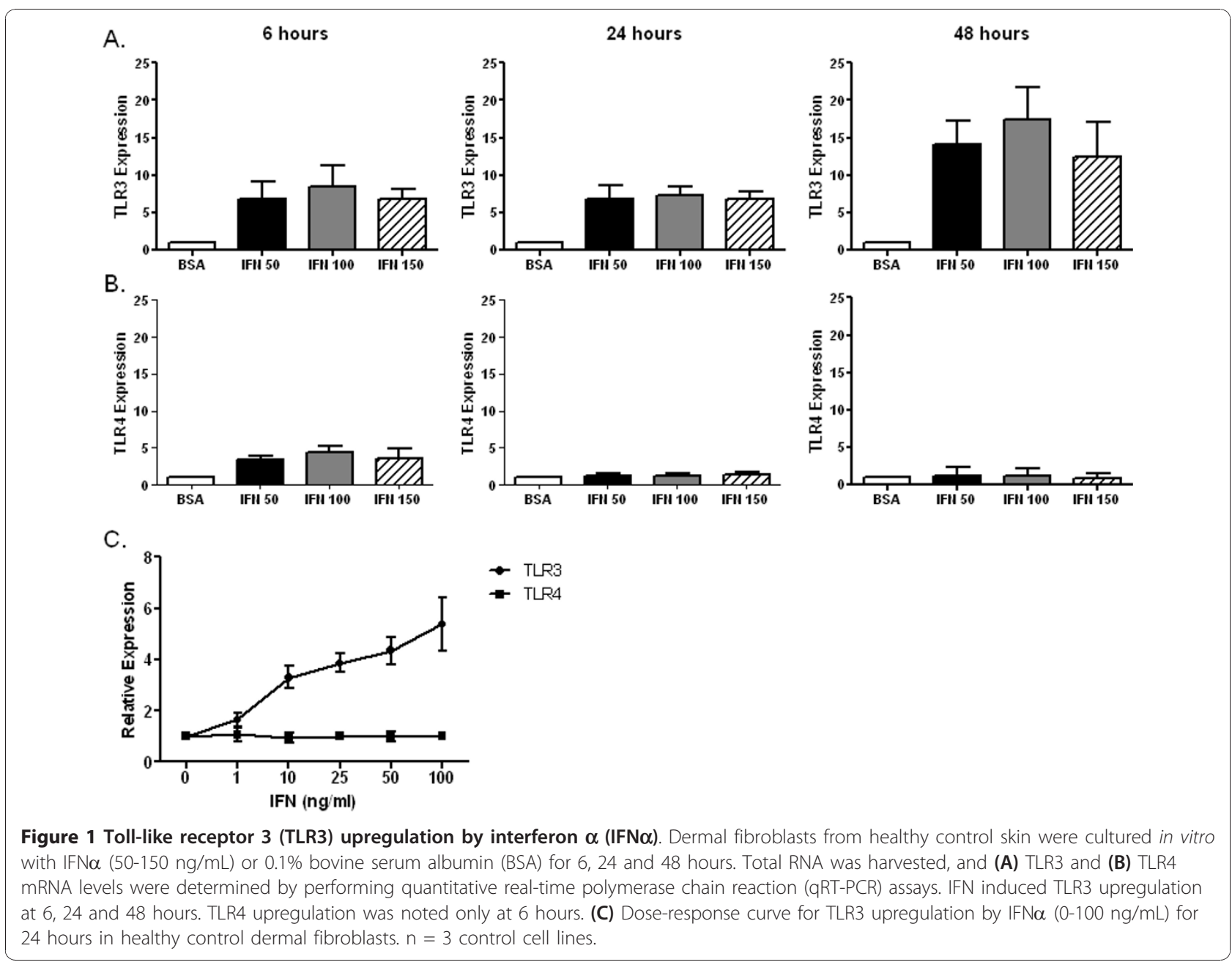

A.

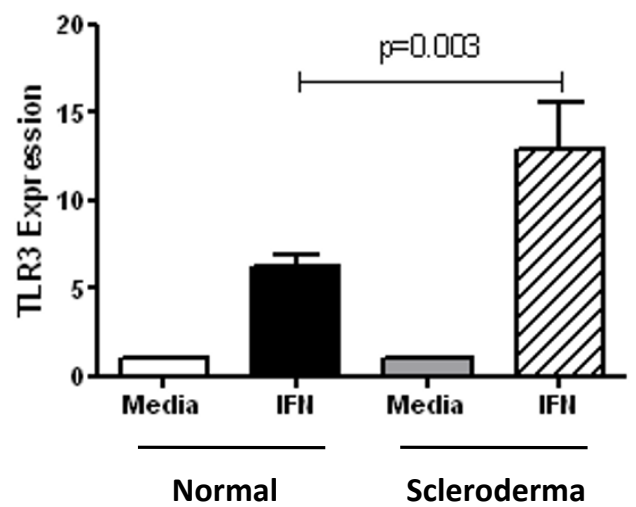

B.

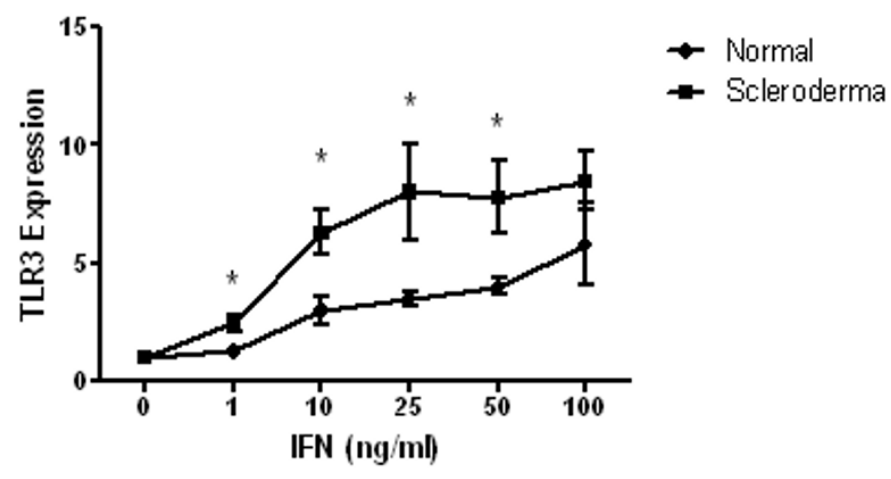

Figure 2 Comparison of TLR3 upregulation by IFN $\alpha$ in healthy control and systemic sclerosis (SSc), or scleroderma, dermal fibroblasts (A) Dermal fibroblasts were stimulated for 24 hours with $50 \mathrm{ng} / \mathrm{mL} I F N \alpha$, and TLR3 was determined by performing qRT-PCR assays. The magnitude of induction of TLR3 expression by IFN $\alpha$ was significantly greater in dermal fibroblasts from patients with SSC $(n=11)$ than in those from healthy controls ( $n=25 ; P=0.003$ ). (B) SSC dermal fibroblasts have a greater magnitude of upregulation of TLR3 with IFN at concentrations ranging from 1 to $100 \mathrm{ng} / \mathrm{mL}\left(\mathrm{n}=4\right.$ in each group; ${ }^{*} P<0.05$ (Wilcoxon signed-rank test)). 
A.

\section{Normal Derm al Fibroblast}

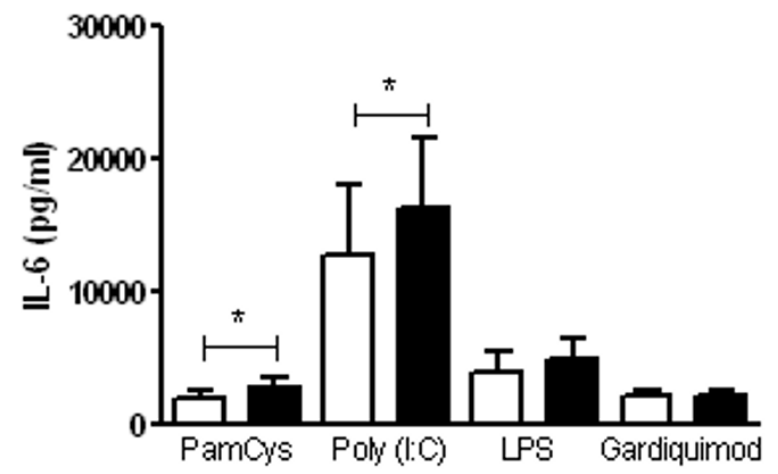

B.

Scleroderma Dermal Fibroblasts

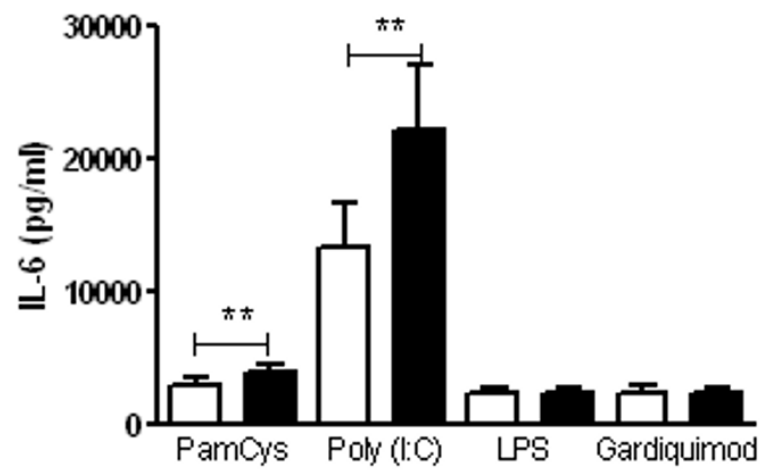

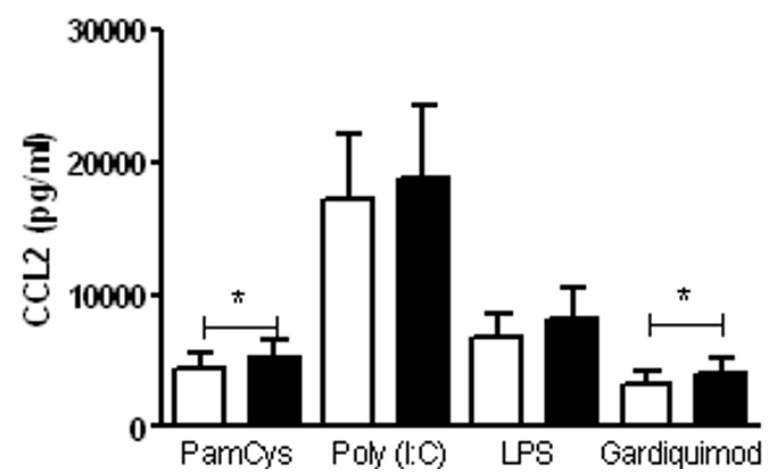

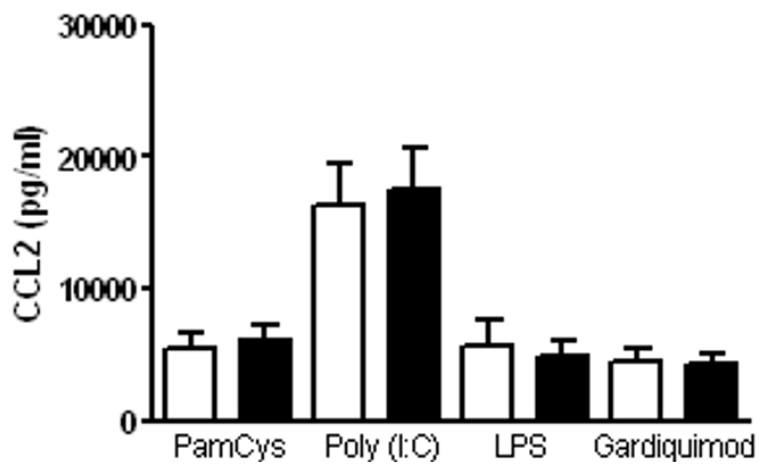

Figure 3 IFN increases TLR3-induced interleukin (IL)-6 production in cultured dermal fibroblasts. (A) Healthy control fibroblasts $(n=10)$ and (B) SSc dermal fibroblasts $(n=10)$ were preincubated with media alone or with $50 \mathrm{ng} / \mathrm{mL}$ IFNa for 24 hours, washed and then stimulated with $\mathrm{Pam}_{3} \mathrm{CysK}_{4}$ (TLR2 agonist); polyinosinic:polycytidylic acid, or poly(l:C) (TLR3 agonist); lipopolysaccharide (TLR4 agonist) and Gardiquimod (TLR7/8 agonist; [1-(4-amino-2-ethylaminomethylimidazo[4,5-c]quinolin-1-y)-2-methylpropan-2-ol]) for 48 hours (10 $\mu \mathrm{g} / \mathrm{mL})$. Culture supernatants were assessed for IL-6 and CC chemokine ligand 2 (CCL2). Preincubation with IFNa increased poly(I:C)-stimulated IL-6 but not CCL2 production from healthy control and SSC dermal fibroblasts. ${ }^{*} P<0.05$, ${ }^{*} P<0.01$ (Wilcoxon signed-rank test).

not shown). These data demonstrate that IFN $\alpha 2$ specifically upregulates TLR3 expression in dermal fibroblasts, which results in increased IL-6 production upon TLR3 stimulation of dermal fibroblasts.

\section{Myofibroblasts have increased upregulation of TLR3}

SSc skin biopsies have increased numbers of myofibroblasts [3]. In vitro TGF- $\beta$ induces the differentiation from fibroblasts to myofibroblasts [21]. Since SSc fibroblasts have an increased induction of TLR3 by IFN 22 compared with control fibroblasts, we sought to determine whether IFN 22 induction of TLR3 expression was increased in myofibroblasts.

Control dermal fibroblasts were cultured in TGF- $\beta$ for 72 hours to induce myofibroblast differentiation in vitro, followed by stimulation with IFNa 2 for 24 hours. As expected, TGF- $\beta$ increased the number of cultured fibroblasts expressing $\alpha$-smooth muscle actin as detected using immunofluoresence (data not shown). Interestingly, dermal fibroblasts preincubated with TGF$\beta$ had greater induction of TLR3 by IFN $\alpha 2$ compared with fibroblasts preincubated in media alone (14.83 \pm 2.06 vs. $7.46 \pm 1.62 ; P=0.02$ ) (Figure $4 \mathrm{~A}$ ). In contrast, dermal fibroblasts preincubated with TGF- $\beta$ had a decrease in TLR4 induction by IFN 2 compared with fibroblasts preincubated in media alone $(1.1 \pm 0.1$ vs. 1.6 $\pm 0.1 ; P=0.001)$. Therefore, myofibroblasts display increased upregulation of TLR3 in response to IFN 22.

\section{Coincubation of IFN $\alpha 2$ and TGF- $\beta$}

Multiple lines of evidence point to the dysregulation of TGF- $\beta$ and IFN $\alpha 2$ in SSc $[12,22]$. How these two cytokines interact at the level of the dermal fibroblasts has not been fully elucidated. TGF- $\beta$ has profibrotic properties, while previous studies have suggested that IFN may have antifibrotic properties. It is reasonable to 


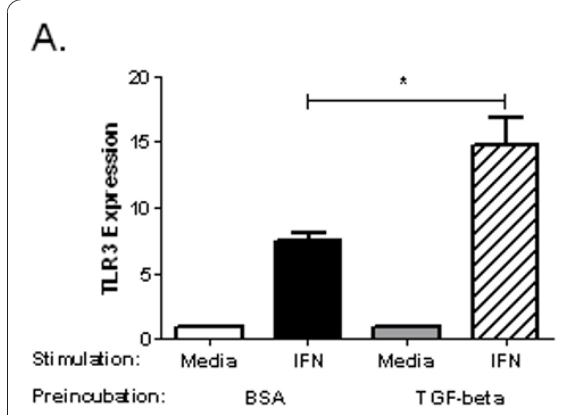

B. Normal Fibroblasts
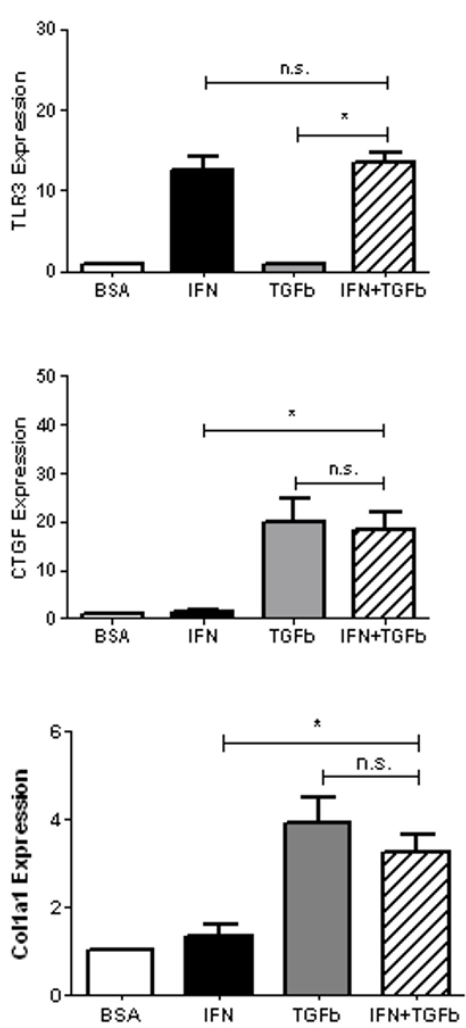

Scleroderma Fibroblasts
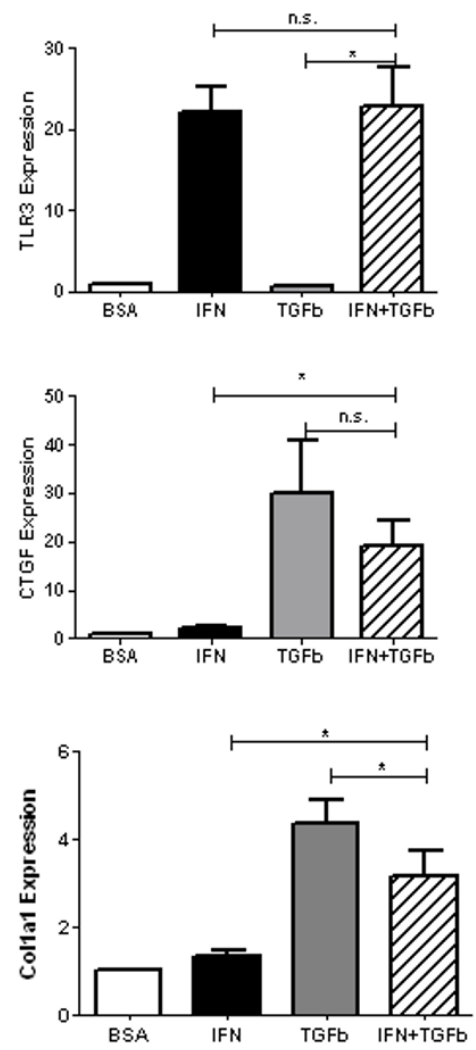

Figure 4 Cross-regulation of IFN $\alpha$ and TGF $\beta$ in dermal fibroblasts. (A) Healthy control dermal fibroblasts were cultured in $10 \mathrm{ng} / \mathrm{mL}$ TGF $\beta$ for 72 hours to induce myofibroblast differentiation in vitro. After 72 hours, cultures were washed and subsequently stimulated with $50 \mathrm{ng} / \mathrm{mL}$ IFN for 24 hours. Total RNA was analyzed for TLR3 by qRT-PCR assay. Preincubation with TGF $\beta$ resulted in a greater induction of TLR3 by IFN compared with fibroblasts preincubated in $0.1 \% \mathrm{BSA}(\mathrm{n}=7 ; P=0.02$ ). (B) Dermal fibroblasts were incubated with $50 \mathrm{ng} / \mathrm{mL} \mathrm{IFN}, 10 \mathrm{ng} / \mathrm{mL} \mathrm{TGF}-$ $\beta$ or both cytokines for 24 hours. Total RNA was analyzed for TLR3, connective tissue growth factor (CTGF), and collagen type I, $\alpha_{1}$ (COL1A1) expression by qRT-PCR assay. Coincubation of fibroblasts with IFN and TGF- $\beta$ did not alter the expression of TLR3 compared with IFN alone. IFN did not alter TGF- $\beta$-induced CTGF expression but did slightly reduce COL1A1 expression in SSC dermal fibroblasts. $\mathrm{n}=7,{ }^{*} P<0.05$, n.s. $=$ not significant (Wilcoxon signed-rank test).

hypothesize that dermal fibroblasts might be exposed simultaneously to both IFN $\alpha 2$ and TGF- $\beta$ in vivo. Therefore, we next sought to ascertain the effects of the IFN $\alpha 2$-induced TLR3 upregulation during simultaneous exposure to TGF- $\beta$.

Fibroblasts were incubated with IFN $\alpha 2$, TGF- $\beta$ or both cytokines for 24 hours. Total RNA was harvested for qRT-PCR analysis (Figure 4B). TLR3 expression was increased by IFN $\alpha 2$ in both control and SSc fibroblasts. Coincubation of fibroblasts with IFN $\alpha 2$ and TGF- $\beta$ did not change the expression of TLR3 compared with IFN $\alpha 2$ alone. CTGF and type I collagen expression also were assessed to determine whether concentrations of IFNa2 that induced TLR3 have antifibrotic properties. CTGF expression was increased by TGF- $\beta$ in both control and SSc fibroblasts $(20.04 \pm 4.6$ and $30.13 \pm 10.62$, respectively). IFN 22 resulted in a slight nonsignificant decrease in TGF- $\beta$-stimulated CTGF expression in both control and SSc fibroblasts $(18.27 \pm 3.9$ and $19.17 \pm$ 2.58 , respectively). Furthermore, collagen, type I, $\alpha_{1}$ (COL1A1) expression was increased by TGF- $\beta$ in both healthy control and SSc fibroblasts $(3.90 \pm 0.60$ and 4.34 \pm 0.58 , respectively). IFN $\alpha 2$ resulted in a slight decrease in COL1A1 expression in both control and SSc fibroblasts; however, this difference was significant only in the SSc fibroblasts $(3.25 \pm 0.41$ and $3.13 \pm 0.58$, respectively). The expression of CTGF and COL1A1 was significantly higher in dermal fibroblasts stimulated with both IFN $\alpha 2$ and TGF- $\beta$ compared with media or IFN $\alpha 2$ alone, suggesting that IFN $\alpha 2$ only blunted the TGF- $\beta$ induction of CTGF and COL1A1. These data suggest that IFNa 2 may decrease expression of matrix-related genes important in the development of dermal fibrosis; however, at concentrations that induce TLR3 expression, 
the magnitude of inhibition is relatively small compared with the overall induction by TGF- $\beta$ alone.

\section{TLR3 expression in fibrotic and scleroderma skin}

The data above were obtained using cultured dermal fibroblasts. To determine whether TLR expression is also found in the fibroblasts in vivo, immunohistochemical studies were performed to localize the expression of TLR3 in skin from the bleomycin-induced skin fibrosis model (Figure 5A), as well as from the skin biopsies of healthy controls and patients with SSc (Figure 5B).

Skin biopsies were performed on mice injected daily for 28 days with subcutaneous saline or bleomycin. Staining with an antibody specific for TLR3 did not reveal any detectable level of TLR3 expression in salineinjected skin (Figure 5A, histograms a and b). In contrast, skin biopsies from mice injected with bleomycin demonstrated expression of TLR3 that was present in cells of the dermis (Figure 5A, histogram d), which localized to fibroblast-like cells (Figure 5A, histogram e) as well as some inflammatory cells (Figure 5A, histogram f). These data demonstrate that TLR3 expression is increased in the dermis of mice injected with bleomycin.

To determine whether TLR3 is expressed in human skin, immunohistochemistry was performed for TLR3 in healthy control skin biopsies and SSc skin biopsies. TLR3 expression was not detectable in the dermis of healthy control skin (Figure 5B, histograms g-i). In contrast, TLR3 expression was observed with higher-power magnification in the dermis of SSc skin (Figure 5B, histogram k) which was localized to fibroblast-like cells as well as inflammatory cells (Figure 5B, histogram l). Last, in SSc skin, the endothelial cells also demonstrated expression of TLR3 (Figure 5B, histogram $\mathrm{m}$ ), which was not observed in healthy control skin biopsies. Therefore, similar to the in vitro data, TLR3 is expressed on fibroblasts in SSc biopsies.

\section{Discussion}

In the current article, we have demonstrated that IFN $\alpha 2$, a type I interferon, increases the expression of TLR3 on human dermal fibroblasts, which results in enhanced TLR3-induced IL-6 production. Dermal fibroblasts from patients with SSc have an augmented response to IFN with regard to TLR3 expression. Consistent with the in vitro data, we also have demonstrated that skin biopsies from patients with SSc as well as the bleomycin-induced skin fibrosis model both have TLR3 expression that localizes to fibroblast-like cells. Importantly, pretreatment with TGF- $\beta$ increased TLR3 induction by IFN $\alpha 2$, but coincubation of TGF- $\beta$ does not alter TLR3 induction by IFN $\alpha 2$. Last, IFN $\alpha 2$ inhibits but does not completely block the induction of CTGF and collagen expression by TGF- $\beta$ in dermal fibroblasts.
TLR3 is a member of the TLR family that recognizes double-stranded RNA, which is a molecular pattern produced by many viruses at some point in their infectious cycle [17]. TLR3 is expressed on endosomes of dendritic cells, but has been reported on the cell surface as well as in endosomes of fibroblasts [17]. Activation of TLR3 results in the production of type I IFN, which may in turn further upregulate the expression of TLR3. With regard to dermal fibroblasts and SSc, the potential TLR3 ligands are unknown. While viral triggers can be considered, there are no consistent associations of SSc with specific viral infections. It is intriguing to hypothesize that complexes of self-RNA andantimicrobial peptides, which have been reported to stimulate TLR7 and TLR8 [23], could also activate TLR3, but this is speculative. One additional hypothesis is that the ECM itself may serve as a TLR3 ligand. Indeed, in addition to PAMPs, TLRs can be activated by damage-associated molecular patterns (DAMPs). DAMPs are proinflammatory molecules generated upon tissue injury that include those released from necrotic cells as well as from the ECM. Tenascin- $\mathrm{C}$ has recently been reported to activate TLR4 during the development of inflammatory arthritis [24]. In the current study, the expression of TLR3 in human skin was demonstrated on dermal fibroblasts within dense connective tissue of the dermis. It is intriguing to hypothesize that the ECM may contain TLR3 ligands that could activate the dermal fibroblasts, even in the absence of a viral trigger.

The function of TLRs is best characterized in the innate immune system, where TLRs signal the presence of an infection and direct the adaptive immune response against microbial antigens [16]. The role of TLR signaling in fibroblasts is not as clearly understood. TLR stimulation of different fibroblast populations has been demonstrated to increase the production of chemokines and cytokines by fibroblasts, which subsequently can increase the inflammatory infiltration of the tissue. In this study, IFN 22 upregulated TLR3 and TLR3-induced IL- 6 production. The increase in IL- 6 could contribute to dermal fibrosis through increased fibroblast survival and proliferation, ECM deposition and myofibroblast differentiation [25-27]. In addition, IL-6 may act synergistically with TGF- $\beta$ with regard to the development of tissue fibrosis [28]. Last, TLR3 activation may also directly regulate the behavior of fibroblasts. A recent report has demonstrated that TLR3 activation with poly (I:C) increased ECM and $\alpha$-smooth muscle actin production, a marker of myofibroblast differentiation, by lung fibroblasts [29]. Together the effects of TLR3 directly on dermal fibroblast ability to differentiate into a myofibroblast and through the production of IL-6 may contribute to the development of dermal fibrosis. 


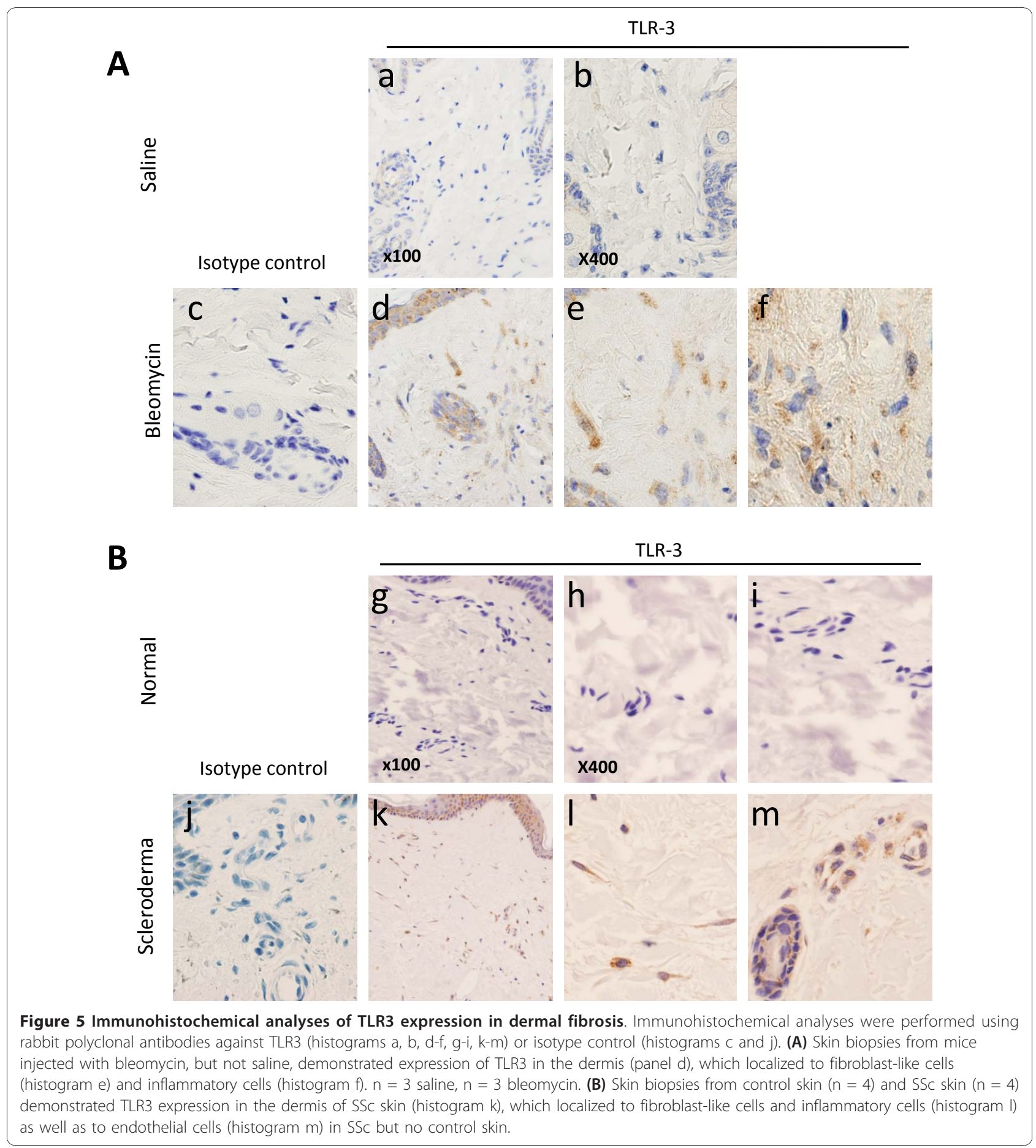

Several independent studies have demonstrated that the type I IFN pathways are upregulated in patients with SSc compared with healthy controls [10-15]. However, the role of type I IFNs in the pathogenesis of SSc remains to be determined. Plasmacytoid dendritic cells (pDCs) are the primary source of type I IFNs in SLE $[10,30]$. It also has been suggested that pDCs are key producers of type I IFNs in SSc [31,32]. Type I IFNs subsequently regulate the behavior of key cells involved in the development of SSc, including dendritic cells, Tcells and dermal fibroblasts. This regulation of dermal fibroblasts could potentially be a pathologic or a protective response. In contrast to Th2 cytokines IL-4 and IL13, which are profibrotic, type II IFNs such as IFN- $\gamma$ 
decrease collagen production by dermal fibroblasts [33-37]. Type I IFNs have also been reported to decrease collagen production by dermal fibroblasts in vitro $[35,36]$. Consistent with the in vitro effects of IFN $\alpha 2$ on collagen production, administration of IFN- $\gamma$ to mice decreased dermal fibrosis and collagen deposition in the bleomycin-induced skin fibrosis model [38]. However, clinical trials of recombinant IFN- $\gamma$ or IFN- $\alpha$ in patients with SSc failed to show substantial clinical benefit [39-41]. The lack of effect of IFNs in SSc may be due to the timing of administration, the particular preparations of IFNs, pharmacokinetics or other clinical reasons. Alternatively, type I IFNs may have additional effects on the behavior of dermal fibroblasts that are independent of their antifibrotic properties.

The data presented herein suggest that type I IFNs may increase the inflammatory potential of the dermal fibroblast in part through the upregulation of TLR3 expression. Furthermore, IFNa2 increases the inflammatory potential more in SSc fibroblasts than in normal fibroblasts. We observed these effects at concentrations as low as $1 \mathrm{ng} / \mathrm{mL}$ IFNa2. The levels of IFNa 2 within the microenvironment of the skin are not known. Therefore, it remains possible that the levels of IFN $\alpha 2$ used in the current study are higher than those found in vivo. At concentrations capable of inducing TLR3 expression, IFN $\alpha 2$ only marginally blunted TGF- $\beta$ induced collagen production, which itself was still significantly elevated relative to unstimulated dermal fibroblasts. Interestingly, it has recently been reported that TLR3 stimulation of dermal fibroblasts increased the expression of IFN $\alpha 2$ - and TGF- $\beta$-responsive genes and that mice treated with subcutaneous TLR3 agonists developed dermal inflammation followed by fibrosis [42]. Together these observations suggest that IFNs may contribute to the development of SSc in a stepwise model wherein the pDCs produce type I IFNs, which regulate not only inflammatory cells but also dermal fibroblasts. Type I IFNs might then increase the expression of a number of molecules on the dermal fibroblast, including TLR3. TLR3 activation, either through viruses or through DAMPs, could increase the inflammatory potential of the dermal fibroblast, including increased IL- 6 production, and could further increase IFN- and TGF- $\beta$-responsive gene expression. Together it is possible that the net balance would ultimately lead to the development of dermal inflammation and fibrosis. In vivo mouse studies will be helpful in determining the overall balance between the antifibrotic and proinflammatory properties of IFNs.

\section{Conclusions}

In summary, our observations suggest that type I IFNs can increase the inflammatory potential of the dermal fibroblast through upregulation of TLR3 and its downstream responses. These studies add to our understanding of how type I IFNs, which are increased in SSc, may contribute to the pathogenesis of SSc. Additional studies are needed to further clarify how type I IFNs may contribute to SSc pathogenesis and to help determine whether type I IFNs can be a rational therapeutic target in SSc.

\section{Abbreviations}

DAMPs: damage-associated molecular patterns; ECM: extracellular matrix; IFN: interferon; SLE: systemic lupus erythematosus; SSc: systemic sclerosis; TLR: Toll-like receptor.

\section{Acknowledgements}

We thank Mei Huang for her assistance with experiments in this manuscript. This study was supported by the Scleroderma Foundation New Investigator Award (SKA), National Institutes of Health/National Institute of Arthritis and Musculoskeletal and Skin Diseases (NIH/NIAMS) grant K08AR054404 (SKA), $\mathrm{NIH/NIAMS}$ Center of Research Translation in Scleroderma grant P50AR054144 (FCA and FKT), and the NIH/NIAMS Scleroderma Family Registry and DNA Repository grant N01-AR-0-2251) (MDM).

\section{Author details}

${ }^{1}$ Division of Rheumatology and Clinical Immunogenetics, Department of Internal Medicine, The University of Texas Health Science Center at Houston, 6431 Fannin Avenue, Houston, TX 77030, USA. ${ }^{2}$ Division of Plastic and Reconstructive Surgery, Department of Surgery, The University of Texas Health Science Center at Houston, 6431 Fannin Avenue, Houston, TX 77030, USA.

\section{Authors' contributions}

SKA, MW and FKT contributed to the study design, data acquisition, data analysis and interpretation, and manuscript preparation. CKL, DHP, MDM and FCA contributed to data acquisition and manuscript preparation.

\section{Competing interests}

The authors declare that they have no competing interests.

Received: 7 September 2010 Revised: 8 December 2010 Accepted: 11 January 2011 Published: 11 January 2011

\section{References}

1. Varga J, Abraham D: Systemic sclerosis: a prototypic multisystem fibrotic disorder. J Clin Invest 2007, 117:557-567.

2. Varga J: Systemic sclerosis: an update. Bull NYU Hosp Jt Dis 2008, 66:198-202.

3. Kissin EY, Merkel PA, Lafyatis R: Myofibroblasts and hyalinized collagen as markers of skin disease in systemic sclerosis. Arthritis Rheum 2006, 54:3655-3660

4. Tomasek JJ, Gabbiani G, Hinz B, Chaponnier C, Brown RA: Myofibroblasts and mechano-regulation of connective tissue remodelling. Nat Rev Mol Cell Biol 2002, 3:349-363.

5. Feghali CA, Bost KL, Boulware DW, Levy LS: Control of IL-6 expression and response in fibroblasts from patients with systemic sclerosis. Autoimmunity 1994, 17:309-318.

6. Suzuki H, Takemura H, Yoshizaki K, Koishihara Y, Ohsugi Y, Okano A, Akiyama Y, Tojo T, Kishimoto T, Kashiwagi H: IL-6-anti-IL-6 autoantibody complexes with IL-6 activity in sera from some patients with systemic sclerosis. J Immunol 1994, 152:935-942.

7. Distler O, Pap T, Kowal-Bielecka O, Meyringer R, Guiducci S, Landthaler M, Schölmerich J, Michel BA, Gay RE, Matucci-Cerinic M, Gay S, MüllerLadner U: Overexpression of monocyte chemoattractant protein 1 in systemic sclerosis: role of platelet-derived growth factor and effects on monocyte chemotaxis and collagen synthesis. Arthritis Rheum 2001, 44:2665-2678.

8. Yamamoto T, Eckes B, Hartmann K, Krieg T: Expression of monocyte chemoattractant protein-1 in the lesional skin of systemic sclerosis. J Dermatol Sci 2001, 26:133-139. 
9. Fleischmajer R, Perlish JS, Reeves JR: Cellular infiltrates in scleroderma skin. Arthritis Rheum 1977, 20:975-984.

10. Blanco P, Palucka AK, Gill M, Pascual V, Banchereau J: Induction of dendritic cell differentiation by IFN- $a$ in systemic lupus erythematosus. Science 2001, 294:1540-1543.

11. Baechler EC, Batliwalla FM, Karypis G, Gaffney PM, Ortmann WA, Espe KJ, Shark KB, Grande WJ, Hughes KM, Kapur V, Gregersen PK, Behrens TW: Interferon-inducible gene expression signature in peripheral blood cells of patients with severe lupus. Proc Natl Acad Sci USA 2003, 100:2610-2615.

12. Tan FK, Zhou X, Mayes MD, Gourh P, Guo X, Marcum C, Jin L, Arnett FC Jr: Signatures of differentially regulated interferon gene expression and vasculotrophism in the peripheral blood cells of systemic sclerosis patients. Rheumatology (Oxford) 2006, 45:694-702.

13. York MR, Nagai T, Mangini AJ, Lemaire R, van Seventer JM, Lafyatis R: A macrophage marker, Siglec-1, is increased on circulating monocytes in patients with systemic sclerosis and induced by type I interferons and toll-like receptor agonists. Arthritis Rheum 2007, 56:1010-1020.

14. Duan $\mathrm{H}$, Fleming J, Pritchard DK, Amon LM, Xue J, Arnett HA, Chen G, Breen P, Buckner JH, Molitor JA, Elkon KB, Schwartz SM: Combined analysis of monocyte and lymphocyte messenger RNA expression with serum protein profiles in patients with scleroderma. Arthritis Rheum 2008, 58:1465-1474

15. Gardner H, Shearstone JR, Bandaru R, Crowell T, Lynes M, Trojanowska M, Pannu J, Smith E, Jablonska S, Blaszczyk M, Tan FK, Mayes MD: Gene profiling of scleroderma skin reveals robust signatures of disease that are imperfectly reflected in the transcript profiles of explanted fibroblasts. Arthritis Rheum 2006, 54:1961-1973.

16. Medzhitov R: Toll-like receptors and innate immunity. Nat Rev Immunol 2001, 1:135-145

17. Matsumoto M, Seya T: TLR3: interferon induction by double-stranded RNA including poly(l:C). Adv Drug Deliv Rev 2008, 60:805-812.

18. Roelofs MF, Wenink MH, Brentano F, Abdollahi-Roodsaz S, OppersWalgreen B, Barrera P, van Riel PL, Joosten LA, Kyburz D, van den Berg WB, Radstake TR: Type I interferons might form the link between Toll-like receptor (TLR) 3/7 and TLR4-mediated synovial inflammation in rheumatoid arthritis (RA). Ann Rheum Dis 2009, 68:1486-1493.

19. Subcommittee for Scleroderma Criteria of the American Rheumatism Association Diagnostic and Therapeutic Criteria Committee: Preliminary criteria for the classification of systemic sclerosis (scleroderma). Arthritis Rheum 1980, 23:581-590.

20. Zhou X, Tan FK, Guo X, Wallis D, Milewicz DM, Xue S, Arnett FC: Small interfering RNA inhibition of SPARC attenuates the profibrotic effect of transforming growth factor $\beta 1$ in cultured normal human fibroblasts. Arthritis Rheum 2005, 52:257-261.

21. Hinz B, Phan SH, Thannickal VJ, Galli A, Bochaton-Piallat ML, Gabbiani G: The myofibroblast: one function, multiple origins. Am J Pathol 2007, 170:1807-1816.

22. Lakos G, Takagawa S, Chen SJ, Ferreira AM, Han G, Masuda K, Wang XJ, DiPietro LA, Varga J: Targeted disruption of TGF- $\beta / S$ mad3 signaling modulates skin fibrosis in a mouse model of scleroderma. Am J Pathol 2004, 165:203-217

23. Ganguly D, Chamilos G, Lande R, Gregorio J, Meller S, Facchinetti V, Homey B, Barrat FJ, Zal T, Gilliet M: Self-RNA-antimicrobial peptide complexes activate human dendritic cells through TLR7 and TLR8. J Exp Med 2009, 206:1983-1994.

24. Midwood K, Sacre S, Piccinini AM, Inglis J, Trebaul A, Chan E, Drexler S, Sofat N, Kashiwagi M, Orend G, Brennan F, Foxwell B: Tenascin-C is an endogenous activator of Toll-like receptor 4 that is essential for maintaining inflammation in arthritic joint disease. Nat Med 2009, 15:774-780.

25. Liu X, Das AM, Seideman J, Griswold D, Afuh CN, Kobayashi T, Abe S, Fang Q, Hashimoto M, Kim H, Wang X, Shen L, Kawasaki S, Rennard Sl: The CC chemokine ligand 2 (CCL2) mediates fibroblast survival through IL-6. Am J Respir Cell Mol Biol 2007, 37:121-128.

26. Ghazizadeh M, Tosa M, Shimizu H, Hyakusoku H, Kawanami O: Functional implications of the IL-6 signaling pathway in keloid pathogenesis. $J$ Invest Dermatol 2007, 127:98-105.

27. Seong GJ, Hong S, Jung SA, Lee JJ, Lim E, Kim SJ, Lee JH: TGF- $\beta$-induced interleukin-6 participates in transdifferentiation of human Tenon's fibroblasts to myofibroblasts. Mol Vis 2009, 15:2123-2128.
28. Yoshida M, Sakuma J, Hayashi S, Abe K, Saito I, Harada S, Sakatani M, Yamamoto S, Matsumoto N, Kaneda Y: A histologically distinctive interstitial pneumonia induced by overexpression of the interleukin 6 , transforming growth factor $\beta 1$, or platelet-derived growth factor $B$ gene. Proc Natl Acad Sci USA 1995, 92:9570-9574.

29. Sugiura H, Ichikawa T, Koarai A, Yanagisawa S, Minakata Y, Matsunaga $K$, Hirano T, Akamatsu K, Ichinose M: Activation of Toll-like receptor 3 augments myofibroblast differentiation. Am J Respir Cell Mol Biol 2009, 40:654-662.

30. Gill MA, Blanco P, Arce E, Pascual V, Banchereau J, Palucka AK: Blood dendritic cells and DC-poietins in systemic lupus erythematosus. Hum Immunol 2002, 63:1172-1180.

31. Kim D, Peck A, Santer D, Patole P, Schwartz SM, Molitor JA, Arnett FC, Elkon KB: Induction of interferon-a by scleroderma sera containing autoantibodies to topoisomerase I: association of higher interferon-a activity with lung fibrosis. Arthritis Rheum 2008, 58:2163-2173.

32. Eloranta ML, Franck-Larsson K, Lovgren T, Kalamajski S, Ronnblom A, Rubin K, Alm GV, Ronnblom L: Type I interferon system activation and association with disease manifestations in systemic sclerosis. Ann Rheum Dis 2010, 69:1396-1402

33. Lakos $G$, Melichian D, Wu M, Varga J: Increased bleomycin-induced skin fibrosis in mice lacking the Th1-specific transcription factor T-bet. Pathobiology 2006, 73:224-237.

34. Aliprantis AO, Wang J, Fathman JW, Lemaire R, Dorfman DM, Lafyatis $R$, Glimcher LH: Transcription factor T-bet regulates skin sclerosis through its function in innate immunity and via IL-13. Proc Natl Acad Sci USA 2007, 104:2827-2830

35. Duncan MR, Berman B: Persistence of a reduced-collagen-producing phenotype in cultured scleroderma fibroblasts after short-term exposure to interferons. J Clin Invest 1987, 79:1318-1324.

36. Jimenez SA, Freundlich B, Rosenbloom J: Selective inhibition of human diploid fibroblast collagen synthesis by interferons. J Clin Invest 1984, 74:1112-1116.

37. Rosenbloom J, Feldman G, Freundlich B, Jimenez SA: Inhibition of excessive scleroderma fibroblast collagen production by recombinant ${ }^{-}$interferon: association with a coordinate decrease in types I and III procollagen messenger RNA levels. Arthritis Rheum 1986, 29:851-856.

38. Yamamoto T, Takagawa S, Kuroda M, Nishioka K: Effect of interferon- $y$ on experimental scleroderma induced by bleomycin. Arch Dermatol Res 2000, 292:362-365.

39. Vlachoyiannopoulos PG, Tsifetaki N, Dimitriou I, Galaris D, Papiris SA, Moutsopoulos HM: Safety and efficacy of recombinant $\gamma$ interferon in the treatment of systemic sclerosis. Ann Rheum Dis 1996, 55:761-768.

40. Black CM, Silman AJ, Herrick Al, Denton CP, Wilson H, Newman J, Pompon L, Shi-Wen X: Interferon-a does not improve outcome at one year in patients with diffuse cutaneous scleroderma: results of a randomized, double-blind, placebo-controlled trial. Arthritis Rheum 1999 42:299-305.

41. Grassegger A, Schuler G, Hessenberger G, Walder-Hantich B, Jabkowski J, MacHeiner W, Salmhofer W, Zahel B, Pinter G, Herold M, Klein G, Fritsch PO: Interferon- $\gamma$ in the treatment of systemic sclerosis: a randomized controlled multicentre trial. Br J Dermatol 1998, 139:639-648.

42. Farina GA, York MR, Di Marzio M, Collins CA, Meller S, Homey B, Rifkin IR, Marshak-Rothstein A, Radstake TR, Lafyatis R: Poly(l:C) drives type I IFNand TGF $\beta$-mediated inflammation and dermal fibrosis simulating altered gene expression in systemic sclerosis. J Invest Dermatol 2010, 130:2583-2593.

doi:10.1186/ar3221

Cite this article as: Agarwal et al:: Toll-like receptor 3 upregulation by type I interferon in healthy and scleroderma dermal fibroblasts. Arthritis Research \& Therapy 2011 13:R3. 